Research report

\title{
Comorbidity variation in patients with obsessive-compulsive disorder according to symptom dimensions: Results from a large multicentre clinical sample
}

\author{
Albina R. Torres ${ }^{\mathrm{a}, *}$, Leonardo F. Fontenelle ${ }^{\mathrm{b}}$, Roseli G. Shavitt ${ }^{\mathrm{c}}$, Ygor A. Ferrão ${ }^{\mathrm{d}}$, \\ Maria Conceição do Rosário $^{\mathrm{e}}$, Eric A. Storch ${ }^{\mathrm{f}}$, Euripedes C. Miguel ${ }^{\mathrm{C}}$ \\ ${ }^{a}$ Department of Neurology, Psychology and Psychiatry, Botucatu Medical School, Univ Estadual Paulista, Brazil \\ ${ }^{\mathrm{b}}$ Anxiety and Depression Research Program, Institute of Psychiatry, Federal University of Rio de Janeiro E' D'Or Institute for Research and Education, Brazil \\ ${ }^{\mathrm{c}}$ Department of Psychiatry, University of São Paulo Medical School, Brazil \\ ${ }^{\mathrm{d}}$ Department of Psychiatry, Health Sciences Federal University of Porto Alegre, Brazil \\ e Child and Adolescent Psychiatry Unit (UPIA) at the Department of Psychiatry, Federal University of São Paulo, Brazil \\ ${ }^{\mathrm{f}}$ Department of Pediatrics and Department of Health Policy and Management, University of South Florida, Rogers Behavioral Health - Tampa Bay, All \\ Children's Hospital - Johns Hopkins Medicine, USA
}

\section{A R T I C L E I N F O}

\section{Article history:}

Received 14 July 2015

Received in revised form

17 September 2015

Accepted 27 October 2015

Available online 30 October 2015

Keywords:

Obsessive-compulsive disorder

Symptom dimensions

Comorbidity

Comorbid disorders

\begin{abstract}
A B S T R A C T
Background: Obsessive-compulsive disorder (OCD) has a heterogeneous and complex phenomenological picture, characterized by different symptom dimensions and comorbid psychiatric disorders, which frequently co-occur or are replaced by others over the illness course. To date, very few studies have investigated the associations between specific OCD symptom dimensions and comorbid disorders. Methods: Cross-sectional, multicenter clinical study with 1001 well-characterized OCD patients recruited within the Brazilian Research Consortium on Obsessive-Compulsive and Related Disorders. The primary instruments were the Dimensional Yale-Brown Obsessive Compulsive Scale (DY-BOCS) and the Structured Clinical Interview for DSM-IV Axis I Disorders. Bivariate analyses between symptom dimensions and comorbidities were followed by logistic regression.

Results: The most common comorbidities among participants ( $56.8 \%$ females) were major depression (56.4\%), social phobia (34.6\%), generalized anxiety disorder (34.3\%), and specific phobia (31.4\%). The aggressive dimension was independently associated with posttraumatic stress disorder (PTSD), separation anxiety disorder, any impulse-control disorder and skin picking; the sexual-religious dimension was associated with mood disorders, panic disorder/agoraphobia, social phobia, separation anxiety disorder, non-paraphilic sexual disorder, any somatoform disorder, body dysmorphic disorder and tic disorders; the contamination-cleaning dimension was related to hypochondriasis; and the hoarding dimension was associated with depressive disorders, specific phobia, PTSD, impulse control disorders (compulsive buying, skin picking, internet use), ADHD and tic disorders. The symmetry-ordering dimension was not independently associated with any comorbidity.

Limitations: Cross-sectional design; participants from only tertiary mental health services; personality disorders not investigated.

Conclusions: Different OCD dimensions presented some specific associations with comorbid disorders, which may influence treatment seeking behaviors and response, and be suggestive of different underlying pathogenic mechanisms.
\end{abstract}

(c) 2015 Elsevier B.V. All rights reserved.

\section{Introduction}

Obsessive-compulsive disorder $(\mathrm{OCD})$ is a heterogeneous

\footnotetext{
* Correspondence to: Departamento de Neurologia, Psicologia e Psiquiatria, Faculdade de Medicina de Botucatu (FMB) - UNESP, Av. Prof. Montenegro s/n, Distrito de Rubião Jr., Botucatu, SP 18.618-970, Brazil.

E-mail addresses: torresar@fmb.unesp.br, albinatorres@gmail.com (A.R. Torres).
}

neuropsychiatric condition that is frequently comorbid with other psychiatric disorders, both in community-based (Torres et al., 2006; Ruscio et al., 2010; Adam et al., 2012; Fineberg et al., 2013) and clinical samples (Tükel et al., 2002; LaSalle et al., 2004; Kalra et al., 2008; Hofmeijer-Sevink et al., 2013).). These complicated psychopathological manifestations pose a challenge for researchers and clinicians, but understanding and dealing with this complex phenomenological picture is a necessity, since potentially 
important etiological and therapeutic aspects are involved.

Efforts have been made to investigate the phenotype of OCD as a function of obsessive-compulsive symptom dimensions. Most studies conducted factor analyses of the Yale-Brown ObsessiveCompulsive Scale symptom checklist (Baer, 1994; Leckman et al., 1997; Mataix-Cols et al., 1999, 2002; Denys et al., 2004; Hasler et al., 2005) and have consistently identified four or five factors/ dimensions: (1) symmetry obsessions, and ordering, repeating and counting compulsions; (2) aggressive obsessions and related compulsions; (3) sexual, religious and moral obsessions ('taboo', unacceptable or forbidden thoughts, sometimes in association with aggressive obsessions) and related compulsions; (4) contamination obsessions and cleaning/washing compulsions; and (5) hoarding obsessions and compulsions (Mataix-Cols et al., 2005; Bloch et al., 2008).

Although not mutually exclusive, OCD symptom dimensions exhibit temporal stability (Mataix-Cols et al., 2002; Rufer et al., 2005; Fullana et al., 2007; De la Cruz et al., 2013), partially distinct neuropsychological profiles (Mataix-Cols et al., 2004; McGuire et al., 2014), genetic patterns (Hasler et al., 2007; Chacon et al., 2007), structural and functional neuroimaging correlates (MataixCols et al., 2004; van den Heuvel et al., 2009; Harrison et al., 2013; Gomes de Alvarenga et al., 2012; Via et al., 2014), help-seeking behaviors (Fullana et al., 2009) and response to treatment (MataixCols et al., 1999; Landeros-Weisenberger et al., 2010). Thus, new instruments, such as the Dimensional Obsessive-Compulsive Scale (DY-BOCS; Rosario-Campos et al., 2006), have been developed to effectively assess the occurrence and severity of specific symptom dimensions to further elucidate associations with clinical and etiological characteristics.

The prevalence of psychiatric comorbidities among people with OCD varies widely, depending on methodological considerations, such as type of participants (adults vs. children/adolescents; community vs. treatment-seeking samples), assessment instruments (screening vs. diagnostic tools), expertize of the interviewers (lay vs. mental health professionals) and period of evaluation (current vs. lifetime). The lifetime prevalence estimates of comorbidity in OCD clinical and community samples usually vary from more than 60\% (Tükel et al., 2002; Torres et al., 2006; Kalra et al., 2008; De Mathis et al., 2013; ) to around 90\% (LaSalle et al., 2004; Ruscio et al., 2010). The disorders that most commonly cooccur with OCD are depressive disorders (major depression and dysthymic disorder) and anxiety disorders (generalized anxiety disorder, social anxiety disorder, panic disorder/agoraphobia, specific phobia, separation anxiety disorder) (Pigott et al., 1994; Tükel et al., 2002; LaSalle et al., 2004; Torres et al., 2006; Kalra et al., 2008; Ruscio et al., 2010; Lochner et al., 2014). Nevertheless, obsessive-compulsive spectrum disorders (e.g., body dysmorphic disorder [BDD], trichotillomania, skin picking, tic disorders) (Pigott et al., 1994; Lochner et al., 2014), eating disorders (Pigott et al., 1994), bipolar disorder (Adam et al., 2012), impulse-control and substance use disorders are also described, the latter particularly in community-based samples (Torres et al., 2006; Ruscio et al., 2010; Adam et al., 2012).

Some comorbid disorders may present common causal pathways. For example, childhood sexual abuse has been associated with several psychiatric disorders, including depression, PTSD, borderline personality disorder, eating, panic and somatization disorders, and OCD (Caspi et al., 2008). Moreover, the incidence of comorbidities in OCD seems to vary according to the age at OCD onset and the time sequence of previous comorbidities. For instance, Janowitz et al. (2009) described that, compared to patients with late onset, those with early onset ( $\leq 10$ years) were more likely to present comorbid obsessive-compulsive spectrum disorders, particularly tic disorders. De Mathis et al. (2013) observed that patients with OCD who initially presented with separation anxiety disorder were more likely to develop anxiety, somatoform disorders and PTSD later in life; those with ADHD as the initial occurring diagnosis were more likely to develop substance use disorders; and patients presenting initially with tic disorders were more likely to have trichotillomania, skin picking and BDD. Developmentally, these findings suggest that OCD does not behave as a unitary disorder, but rather as a constellation of symptoms or dimensions that interact with additional psychopathology increasing the vulnerability for subsequent disorders.

The relevance of psychiatric comorbidity in patients with OCD has been related to a number of clinical features, including treatment-seeking behaviors (Torres et al., 2006; Fineberg et al., 2013), clinical severity, chronicity and impairment (Tükel et al., 2002; Fineberg et al., 2013; Jakubovski et al., 2013; Hofmeijer-Sevink et al., 2013),), suicidality (Torres et al., 2011), and treatment response (Ferrão et al., 2006; Raffin et al., 2009; Borges et al., 2011; Jakubovski et al., 2013). Nevertheless, very few studies have examined the specific associations between different OCD symptom dimensions and comorbid disorders (Hasler et al., 2005, 2007), except for the hoarding dimension (Fontenelle et al., 2004; Hartl et al., 2005; LaSalle-Ricci et al., 2006; Wheaton et al., 2008; Frost et al., 2011; Chakraborty et al., 2012; Hall et al., 2013; Samuels et al., 2014). Hasler et al. (2005) evaluated 317 OCD patients and described that aggressive, sexual, religious and somatic obsessions, and checking compulsions were broadly associated with anxiety disorders and depression; obsessions of symmetry and ordering/ arranging, repeating and counting compulsions with panic disorder/agoraphobia and bipolar disorders; and contamination obsessions and cleaning compulsions, with eating disorders. In a study of 418 participants (Hasler et al., 2007), aggressive, sexual and religious obsessions, and checking compulsions were linked to affective disorders, whereas symmetry, ordering and arranging compulsions were associated with attention deficit hyperactivity disorder (ADHD), alcohol dependence and bulimia nervosa. The symmetry-ordering dimension has also been previously associated with tic disorders (Miguel et al., 1997; Coffey et al., 1998), whereas the hoarding dimension has been linked to depressive (Winsberg et al., 1999; Coles et al., 2003; Frost et al., 2011; Tolin and Villavicencio, 2011; Hall et al., 2013) and bipolar disorders (Fontenelle et al., 2004; LaSalle-Ricci et al., 2006; Samuels et al., 2007; Wheaton et al., 2008; Chakraborty et al., 2012), generalized anxiety disorder (GAD; Samuels et al., 2007; Chakraborty et al., 2012), panic disorder (Storch et al., 2007; Samuels et al., 2014), specific phobia (Samuels et al., 2014), social phobia (Coles et al., 2003; Samuels et al., 2007; Wheaton et al., 2008), BDD (LaSalle-Ricci et al., 2006; Samuels et al., 2007), substance use disorders (Wheaton et al., 2008), tic disorders (Samuels et al., 2007; 2014), ADHD (Hartl et al., 2005; Tolin and Villavicencio, 2011, Hacker et al., 2012; Frank et al., 2014), posttraumatic stress disorder (PTSD; LaSalle-Ricci et al., 2006) and impulse-control disorders (ICD; Winsberg et al., 1999; Grisham et al., 2007; Tolin and Villavicencio, 2011; Timpano et al., 2014), particularly "grooming behaviors" (Samuels et al., 2002, 2007), compulsive buying (Winsberg et al., 1999; Mueller et al., 2009; Frost et al., 2009, 2011; Bulli et al., 2014) and kleptomania (Frost et al., 2011).

Despite the relevant contributions of these above studies, several methodological limitations exist. In particular, past studies have involved smaller samples, from developed countries, and have not used a detailed, direct and psychometrically sound measure of obsessive-compulsive symptom dimensions (e.g., DYBOCS). Only two studies (Hasler et al., 2005, 2007) examined several dimensions regarding comorbidities. Therefore, despite the heterogeneity of OCD and the high level of comorbidity with other psychiatric disorders, the literature on specific comorbidities as independent predictors of symptom dimensions (i.e., adjusting for the occurrence of other symptom dimensions) is limited. 
Therefore, this large clinical study aims to fill this literature gap, which has potentially important etiological and clinical implications, including impact on treatment seeking, adherence and response. With this in mind, this study investigated the independent associations between each specific OCD symptom dimension and a wide range of lifetime psychiatric comorbidities, in a large multicentre clinical sample.

Our main hypotheses, based on the existing literature, were: there will be an association between: (a) aggressive and/or sexualreligious dimensions and affective/anxiety disorders; (b) symmetry-ordering dimension and bipolar disorder, panic/ agoraphobia, ADHD and tic disorders; (c) contamination-cleaning dimension and eating disorders; and (d) hoarding dimension and affective/anxiety disorders, BDD, substance use, tic disorders, ADHD, PTSD and impulse-control disorders.

\section{Methods}

\subsection{Subjects}

Participants included 1001 OCD patients from the Brazilian Research Consortium on Obsessive-Compulsive Spectrum Disorders (CTOC), recruited from seven public university hospitals specialized outpatient clinics. The DSM-IV OCD diagnosis was confirmed using the Structured Clinical Interview for DSM-IV Axis I disorders (SCID-I; First et al., 1995). Exclusion criteria included psychotic and organic disorders that would impair the understanding of the research questions $(N=8)$ and refusal to participate $(N=40)$. All patients were interviewed face-to-face by experienced and trained clinicians (postgraduate psychologists or psychiatrists) between 2003 and 2009. Each patient's evaluation lasted, on average, five hours. To assess inter-rater reliability, the leaders of each center watched and independently rated at least six patients' videotaped interviews of the SCID and DY-BOCS. Of the 191 analyzed items, 184 (96\%) were concordant by the eight leaders, three items (1.6\%) were discordant for one rater, three items $(1.6 \%)$ for two raters and one item $(0.5 \%)$ for four raters. A more detailed description of the CTOC methods (all assessment instruments and training process) can be found in Miguel et al. (2008).

\subsection{Assessment instruments}

A wide range of structured instruments was used in the complete research assessment; the ones used in the present report are briefly described below:

(1) Dimensional Yale-Brown Obsessive-Compulsive Scale (DYBOCS; Rosario-Campos et al., 2006), to evaluate the presence and severity of OCD symptom dimensions. The DY-BOCS checklist has 88-items divided into six dimensions: harmaggression, sexual-religious, contamination-cleaning, symmetry-ordering-repeating-counting, hoarding and miscellaneous (residual category). The clinical severity of each dimension (both current and previous/worst ever) is evaluated in terms of frequency, distress and interference, with a maximum score of 15, five for each aspect. It also provides an assessment of the overall severity of $O C D$, which ranges from 0 to 15 for symptoms and 0-15 for the associated overall impairment. Checking and repetitive compulsions, avoidant behaviors, and mental rituals are investigated within each dimension. Therefore, whereas the Y-BOCS assesses the severity of (grouped) obsessions and compulsions, the DY-BOCS, by means of a more comprehensive symptom checklist, evaluates obsessions, compulsions and avoidance grouped by their specific content into different dimensions. Only the categorical outcomes (lifetime occurrence of each symptom dimension, except miscellaneous) were analyzed in this study, to be consistent with the lifetime evaluation of comorbid disorders.

(2) Yale-Brown Obsessive-Compulsive Scale (Y-BOCS; Goodman et al., 1989), to evaluate the severity of obsessions and compulsions. The Y-BOCS has 10 items and a maximum score of 40, 20 for obsessions and 20 for compulsions.

(3) The Structured Clinical Interview for DSM-IV Axis I Disorders (SCID-I; First et al., 1995), to evaluate lifetime axis I comorbid conditions and to confirm OCD diagnosis. Additional modules for impulse-control disorders (SCID-TCIm) were also used (First, 2004).

\subsection{Ethical aspects}

The Ethics Committees of all CTOC University Hospitals approved the research protocol. All subjects provided written informed consent after a review of study procedures. Participants were assured that study participation was voluntary and, in case they decided not to participate, this would have no interference whatsoever with the continuity of their treatment. Children and adolescents (4.6\%) were also asked to provide written informed assent in addition to parental consent. Participants did not receive any financial compensation.

\subsection{Statistical analysis}

Categorical data were described as absolute $(n)$ and relative values (\%), and continuous data as mean and standard deviation (SD) or median (range), according to variable distribution. After descriptive analysis, the associations among lifetime occurrence of specific OCD dimensions according to the DY-BOCS (aggressive, sexual-religious, symmetry-ordering, contamination-cleaning and hoarding) and lifetime comorbid psychiatric disorders were examined. The miscellaneous dimension was not included, given its heterogeneous content. Results of bivariate analyses are presented as odds ratios (OR) with 95\% confidence intervals (CI). Given that OCD symptom dimensions frequently co-occur and are not mutually exclusive, logistic regression models were constructed, to adjust each specific symptom dimension-comorbid disorder association that was significant in the bivariate analysis for sex, age and lifetime occurrence of other dimensions. Thus, sex, age and the four other dimensions were included in the regression models, to estimate adjusted odds ratios (AOR) with 95\% confidence intervals. Given the limited data reported to date on the association between symptom dimensions and comorbidity, the statistical significance was set at 0.05 . All analyses were conducted using the STATA 12.0 software (Stata Corporation, 2011).

\section{Results}

The sociodemographic and clinical characteristics of the sample, including symptoms severity, are presented in Table 1 . Most patients were female $(n=569 ; 56.8 \%)$, Caucasian $(n=832 ; 83.1 \%)$ and single $(n=544 ; 54.3 \%)$. The participants' mean age was 34.85 years $(S D=12.99$ ), ranging from 9 to 82 years (only $4.6 \%$ had less than 18 years of age). The most frequently reported dimension was symmetry-ordering $(n=868 ; 86.8 \%)$, followed by contaminationcleaning $(n=737 ; 73.6 \%)$, aggressive $(n=672 ; 67.1 \%)$, sexual-religious ( $n=572 ; 57.1 \%)$ and hoarding $(n=528 ; 52.7 \%)$. Data on the prevalence of comorbid psychiatric disorders are presented in Table 2. As a group, anxiety and mood disorders were the most frequent conditions $(n=699 ; 69.8 \%$ and $n=609 ; 60.8 \%$, respectively). The most common individual comorbidity was major 
Table 1

Sociodemographic and clinical characteristics of the obsessive-compulsive disorder sample $(n=1001)$.

\begin{tabular}{|c|c|c|}
\hline $\begin{array}{l}\text { Characteristic } \\
\text { Categorical variables }\end{array}$ & $\boldsymbol{n}$ & $\%$ \\
\hline \multicolumn{3}{|l|}{ Sex } \\
\hline Female & 569 & 56.8 \\
\hline Male & 432 & 43.2 \\
\hline \multicolumn{3}{|l|}{ Ethnicity } \\
\hline Caucasian & 832 & 83.1 \\
\hline Black/African & 39 & 3.9 \\
\hline Asian & 13 & 1.3 \\
\hline Mixed (white/black) & 115 & 11.5 \\
\hline Other & 2 & 0.2 \\
\hline \multicolumn{3}{|l|}{ Marital status } \\
\hline Single & 544 & 54.3 \\
\hline Married-partnered (e.g, living together) & 377 & 37.7 \\
\hline Divorced-separated & 66 & 6.6 \\
\hline Widowed & 14 & 1.4 \\
\hline \multicolumn{3}{|l|}{ Employment status } \\
\hline Working outside the home & 433 & 43.3 \\
\hline Retired & 108 & 10.8 \\
\hline Student & 179 & 17.9 \\
\hline Unemployed & 154 & 15.4 \\
\hline Working inside the home & 104 & 10.4 \\
\hline Other & 23 & 2.3 \\
\hline \multicolumn{3}{|l|}{ Treatment } \\
\hline Current medication $^{\mathrm{a}}$ & 563 & 56.2 \\
\hline Any history of psychotherapy & 643 & 64.2 \\
\hline Quantitative variables & Mean (SD) & Median (range) \\
\hline Age (in years) & 34.85 (12.99) & $32(9-82)$ \\
\hline Age of OC symptoms onset (in years) & $12.56(7.28)$ & $10(3-54)$ \\
\hline Age of OCD onset (in years) & $21.76(10.63)$ & $19(4-64)$ \\
\hline Educational level (in years) ${ }^{\mathrm{b}}$ & $14.57(4.97)$ & $14(1-31)$ \\
\hline \multicolumn{3}{|l|}{ Y-BOCS-current severity } \\
\hline Obsessions & $12.68(3.94)$ & $13(0-20)$ \\
\hline Compulsions & $12.83(4.14)$ & $13(0-20)$ \\
\hline Total score & $25.51(7.51)$ & $26(7-40)$ \\
\hline \multicolumn{3}{|l|}{ DY-BOCS-current severity } \\
\hline Aggression & $5.29(4.98)$ & $5(0-15)$ \\
\hline Sexual-religious & $4.30(4.90)$ & $2(0-15)$ \\
\hline Symmetry-ordering ${ }^{\mathrm{c}}$ & $7.34(4.64)$ & $8(0-15)$ \\
\hline Contamination-cleaning & $6.22(5.15)$ & $7(0-15)$ \\
\hline Hoarding & $3.15(4.08)$ & $0(0-15)$ \\
\hline Miscellaneous & $7.54(4.70)$ & $9(0-15)$ \\
\hline Total global & $21.15(6.26)$ & $22(0-30)$ \\
\hline
\end{tabular}

$N=$ absolute values; $\%=$ relative values; $\mathrm{SD}=$ standard deviation; YBOCS=YaleBrown Obsessive-Compulsive Scale; DY-BOCS=Dimensional Yale-Brown Obsessive-Compulsive Scale.

a Current medication status includes 397 (39.7\%) subjects on selective serotonin reuptake inhibitors, $102(10.2 \%)$ on clomipramine, $27(2.7 \%)$ on selective serotonin and noradrenaline reuptake inhibitors, which could be used in combination with antipsychotics, benzodiazepines and mood stabilizers.

b $1(0.01 \%)$ missing.

c $2(0.02 \%)$ missing.

depressive disorder $(n=565 ; 56.4 \%$ ), followed by social phobia ( $n=346 ; 34.6 \%)$, generalized anxiety disorder $(n=343 ; 34.3 \%)$ and specific phobia ( $n=314 ; 31.4 \%)$.

The bivariate associations between psychiatric comorbidity and OCD symptom dimensions are presented in Table 3.

Results of the logistic regression models are presented in
Table 2

Prevalence of lifetime comorbid psychiatric disorders in the obsessive-compulsive disorder sample $(N=1001)$.

\begin{tabular}{|c|c|}
\hline Comorbidity & $n(\%)$ \\
\hline Any lifetime comorbid psychiatric disorder & $921(92.1)$ \\
\hline Any mood disorder & $609(60.8)$ \\
\hline Major depressive disorder & $565(56.4)$ \\
\hline Dysthymia & $119(11.9)$ \\
\hline Bipolar I disorder & $38(3.8)$ \\
\hline Any bipolar disorder & $76(7.6)$ \\
\hline Any anxiety disorder & $699(69.8)$ \\
\hline Generalized anxiety disorder & $343(34.3)$ \\
\hline Panic with or without agoraphobia & $202(20.2)$ \\
\hline Social phobia & $346(34.6)$ \\
\hline Specific phobia & $314(31.4)$ \\
\hline Posttraumatic stress disorder & $191(19.1)$ \\
\hline Separation anxiety disorder & $276(27.6)$ \\
\hline Any impulse control disorder & $362(36.2)$ \\
\hline Compulsive buying & $108(10.8)$ \\
\hline Skin-picking & $167(16.7)$ \\
\hline Trichotillomania & $60(6.0)$ \\
\hline Kleptomania & $28(2.8)$ \\
\hline Intermittent explosive disorder & $75(7.5)$ \\
\hline Impulsive-compulsive internet use & $30(3.0)$ \\
\hline Impulsive-compulsive non-paraphilic sexual disorder & $36(3.6)$ \\
\hline Any somatoform disorder & $175(17.5)$ \\
\hline Hypochondriasis & $34(3.4)$ \\
\hline Body dysmorphic disorder & $117(11.7)$ \\
\hline Somatization disorder & $23(2.3)$ \\
\hline Any eating disorder & $114(11.4)$ \\
\hline Anorexia nervosa & $26(2.6)$ \\
\hline Bulimia nervosa & $27(2.7)$ \\
\hline Binge eating disorder & $80(8.0)$ \\
\hline Any alcohol use disorder & $79(7.9)$ \\
\hline Any (illicit) drug use disorder & $35(3.5)$ \\
\hline Attention Deficit Hyperactive Disorder (ADHD) & $137(13.7)$ \\
\hline Any tic disorder & $284(28.4)$ \\
\hline Tourette syndrome & $88(8.8)$ \\
\hline
\end{tabular}

$n=$ absolute values; $\%=$ relative values;

Table 4. The aggressive dimension was independently associated with PTSD, separation anxiety disorder, any impulse-control disorder and skin picking. The sexual-religious dimension was associated with mood disorders, panic disorder/agoraphobia, social phobia, separation anxiety disorder, impulsive-compulsive nonparaphilic sexual disorder, any somatoform disorder, BDD and tic disorders. The contamination-cleaning dimension was associated with hypochondriasis (and negatively associated with Tourette syndrome), and the hoarding dimension was associated with depressive disorders, specific phobia, PTSD, separation anxiety disorder, impulse control disorders (compulsive buying, skin picking, impulsive-compulsive internet use), ADHD and tic disorders. The symmetry-ordering dimension presented no independent comorbid correlates.

\section{Discussion}

OCD presents as phenotypically complex, since at least partially 
Table 3

Psychiatric comorbidities in 1001 OCD patients, according to lifetime occurrence of specific symptom dimensions (bivariate analysis).

\begin{tabular}{|c|c|c|c|c|c|}
\hline & $\begin{array}{l}\text { Aggressive } \\
\text { OR (95\% CI) }\end{array}$ & $\begin{array}{l}\text { Sexual-religious } \\
\text { OR }(95 \% \mathrm{CI})\end{array}$ & $\begin{array}{l}\text { Symmetry-ordering } \\
\text { OR (95\% CI) }\end{array}$ & $\begin{array}{l}\text { Contamination-cleaning } \\
\text { OR }(95 \% \mathrm{CI})\end{array}$ & $\begin{array}{l}\text { Hoarding } \\
\text { OR }(95 \% \mathrm{CI})\end{array}$ \\
\hline Any psychiatric comorbidity & $1.48(1.00-2.19)$ & $1.62(1.10-2.37)$ & ns & ns & ns \\
\hline Any mood disorder & ns & $1.59(1.23-2.05)$ & $1.55(1.07-2.24)$ & ns & $1.80(1.39-2.33)$ \\
\hline Major depression & $1.33(1.02-1.74)$ & $1.44(1.12-1.86)$ & $1.55(1.07-2.39)$ & ns & $1.66(1.29-2.14)$ \\
\hline Dysthymia & $1.98(1.24-3.15)$ & $1.93(1.27-2.94)$ & ns & ns & ns \\
\hline Bipolar I disorder & ns & $2.91(1.32-6.44)$ & ns & ns & ns \\
\hline Any bipolar disorder & ns & ns & ns & ns & ns \\
\hline Any anxiety disorder & $1.39(1.05-1.85)$ & $1.35(1.03-1.77)$ & ns & ns & ns \\
\hline Generalized anxiety disorder & ns & ns & ns & ns & ns \\
\hline Panic disorder with or without Agoraphobia & $1.97(1.30-2.98)$ & $1.63(1.13-2.34)$ & ns & ns & ns \\
\hline Social phobia & ns & $1.45(1.11-1.89)$ & ns & ns & ns \\
\hline Specific phobia & $1.35(1.01-1.82)$ & ns & ns & ns & $1.47(1.11-1.92)$ \\
\hline Posttraumatic stress disorder & $2.24(1.65-3.62)$ & $1.67(1.20-2.34)$ & $1.83(1.06-3.17)$ & ns & $1.86(1.34-2.59)$ \\
\hline Separation anxiety disorder & $1.76(1.29-2.42)$ & $1.63(1.22-2.17)$ & ns & ns & ns \\
\hline Any impulse-control disorder & $1.69(1.27-2.26)$ & $1.36(1.04-1.76)$ & ns & ns & $1.52(1.17-1.98)$ \\
\hline Compulsive buying & ns & ns & ns & ns & $2.21(1.43-3.40)$ \\
\hline Skin picking & $2.33(1.54-3.53)$ & ns & ns & ns & $1.70(1.20-2.39)$ \\
\hline Trichotillomania & ns & ns & ns & ns & ns \\
\hline Kleptomania & ns & ns & ns & ns & $2.29(1.00-5.26)$ \\
\hline Intermittent explosive disorder & $1.88(1.06-3.33)$ & ns & ns & ns & ns \\
\hline Impulsive-compulsive internet use & $4.55(1.36-15.18)$ & ns & ns & ns & $3.03(1.28-7.16)$ \\
\hline Impulsive-compulsive nonparaphilic sexual disorder & ns & $13.49(3.17-57.40)$ & ns & ns & ns \\
\hline Any somatoform disorder & $1.57(1.08-2.27)$ & $1.75(1.24-2.48)$ & ns & ns & ns \\
\hline Hypochondriasis & ns & ns & ns & $3.82(1.15-12.65)$ & ns \\
\hline Body dysmorphic disorder & $1.56(1.00-2.43)$ & $2.39(1.54-3.70)$ & ns & ns & ns \\
\hline Somatization disorder & ns & ns & ns & ns & ns \\
\hline Any eating disorder & ns & $1.51(1.00-2.27)$ & ns & ns & ns \\
\hline Anorexia nervosa & ns & ns & ns & ns & ns \\
\hline Bulimia nervosa & ns & ns & ns & ns & ns \\
\hline Binge eating disorder & ns & $1.72(1.05-2.81)$ & ns & ns & ns \\
\hline Alcohol use disorder & ns & $1.68(1.03-2.76)$ & ns & ns & ns \\
\hline Drug abuse or dependence & ns & ns & ns & ns & ns \\
\hline Attention deficit hyperactive disorder & $1.80(1.18-2.76)$ & $1.64(1.12-2.40)$ & $2.36(1.17-4.78)$ & ns & $2.17(1.47-3.19)$ \\
\hline Any tic disorder & $1.60(1.18-2.19)$ & $1.73(1.30-2.32)$ & $1.72(1.09-2.72)$ & ns & $1.62(1.22-2.15)$ \\
\hline Tourette syndrome & ns & $1.78(1.11-2.85)$ & ns & $0.63(0.39-1.00)$ & ns \\
\hline
\end{tabular}

$\mathrm{ns}=$ non-significant; $\mathrm{OR}=$ Odds ratio; $\mathrm{CI}=$ confidence interval.

different etiological mechanisms seem to be involved in OCD symptom dimensions (Mataix-Cols et al., 2004; Hasler et al., 2007; Chacon et al., 2007; van den Heuvel et al., 2009; Harrison et al., 2013; Gomes de Alvarenga et al., 2012; Via et al., 2014; McGuire et al., 2014), and some comorbidities may present common etiological factors (Cerdá et al., 2008; Caspi et al., 2008). Thus, there are reasonable arguments for both splitting and lumping OCD manifestations. In this study, we investigated the relationship between specific OCD symptom dimensions and comorbid psychiatric conditions in a large and well-characterized clinical sample.

The sexual-religious dimension presented the highest number of associated lifetime comorbidities, followed by hoarding, aggressive and contamination-cleaning dimensions. Our hypotheses were partially supported. In general, the aggressive dimension was associated with PTSD and separation anxiety disorder, any impulse-control disorder and skin picking; the sexual-religious dimension was associated with mood disorders (depressive and bipolar I disorders), anxiety disorders (panic/agoraphobia, social phobia, separation anxiety), non-paraphilic sexual disorder, any somatoform disorder, BDD and tic disorders; the contaminationcleaning dimension was directly associated with hypochondriasis and negatively associated with Tourette syndrome; and the hoarding dimension was directly related to depressive disorders, some anxiety (i.e., specific phobia, PTSD) and impulse-control disorders (i.e., compulsive buying, skin picking, internet use), ADHD and tic disorders.

In most studies, the aggressive dimension was analyzed together with the sexual-religious dimension, due to factor analyses that indicated a single "unacceptable" or "taboo" thoughts dimension. Accordingly, in studies with adult (Grant et al., 2006) and pediatric OCD samples (De la Cruz et al., 2013), individuals presenting sexual obsessions were more likely than those without these obsessions to present with aggressive and religious obsessions. In the present study, common and specific comorbidities of these two dimensions could be identified. Thus, whereas separation anxiety disorder was an independent predictor of both aggressive and sexual-religious dimensions, PTSD and skin picking were only associated with the aggressive dimension. On the other hand, mood disorders, panic disorder/agoraphobia, social phobia, 
Table 4

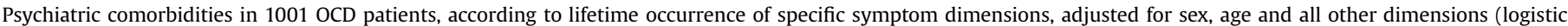
regression).

\begin{tabular}{|c|c|c|c|c|c|}
\hline & $\begin{array}{l}\text { Aggressive } \\
\text { OR (95\% CI) }\end{array}$ & $\begin{array}{l}\text { Sexual-religious } \\
\text { OR }(95 \% \mathrm{CI})\end{array}$ & $\begin{array}{l}\text { Symmetry-ordering } \\
\text { OR (95\% CI) }\end{array}$ & $\begin{array}{l}\text { Contamination-cleaning } \\
\text { OR }(95 \% \mathrm{CI})\end{array}$ & $\begin{array}{l}\text { Hoarding } \\
\text { OR }(95 \% \mathrm{CI})\end{array}$ \\
\hline Any psychiatric comorbidity & ns & ns & ns & ns & ns \\
\hline Any mood disorder & ns & $1.52(1.15-2.02)$ & ns & ns & $1.61(1.23-2.11)$ \\
\hline Major depression & ns & $1.34(1.01-1.77)$ & ns & ns & $1.49(1.14-1.94)$ \\
\hline Dysthymia & ns & $1.68(1.08-2.63)$ & ns & ns & ns \\
\hline Bipolar I disorder & ns & $2.62(1.11-6.17)$ & ns & ns & ns \\
\hline Any bipolar disorder & ns & ns & ns & ns & ns \\
\hline Any anxiety disorder & ns & ns & ns & ns & ns \\
\hline Generalized anxiety disorder & ns & ns & ns & ns & ns \\
\hline Panic disorder with or without Agoraphobia & ns & $1.51(1.06-2.15)$ & ns & ns & ns \\
\hline Social phobia & ns & $1.36(1.02-1.82)$ & ns & ns & ns \\
\hline Specific phobia & ns & ns & ns & ns & $1.39(1.04-1.85)$ \\
\hline Posttraumatic stress disorder & $2.10(1.38-3.22)$ & ns & ns & ns & $1.43(1.01-2.02)$ \\
\hline Separation anxiety disorder & $1.46(1.03-2.05)$ & $1.39(1.02-1.90)$ & ns & ns & ns \\
\hline Any impulse-control disorder & $1.45(1.06-1.99)$ & ns & ns & ns & $1.43(1.08-1.89)$ \\
\hline Compulsive buying & ns & ns & ns & ns & $1.93(1.23-3.05)$ \\
\hline Skin picking & $2.02(1.28-3.17)$ & ns & ns & ns & $1.56(1.08-2.26)$ \\
\hline Trichotillomania & ns & ns & ns & ns & ns \\
\hline Kleptomania & ns & ns & ns & ns & ns \\
\hline Intermittent explosive disorder & ns & ns & ns & ns & ns \\
\hline Impulsive-compulsive internet use & ns & ns & ns & ns & $2.74(1.14-6.61)$ \\
\hline Impulsive-compulsive nonparaphilic sexual disorder & ns & $12.86(2.95-56.12)$ & ns & ns & ns \\
\hline Any somatoform disorder & ns & $1.61(1.10-2.33)$ & ns & ns & ns \\
\hline Hypochondriasis & ns & ns & ns & $4.44(1.31-15.06)$ & ns \\
\hline Body dysmorphic disorder & ns & $2.15(1.35-3.44)$ & ns & ns & ns \\
\hline Somatization disorder & ns & ns & ns & ns & ns \\
\hline Any eating disorder & ns & ns & ns & ns & ns \\
\hline Anorexia nervosa & ns & ns & ns & ns & ns \\
\hline Bulimia nervosa & ns & ns & ns & ns & ns \\
\hline Binge eating disorder & ns & ns & ns & ns & ns \\
\hline Alcohol use disorder & ns & ns & ns & ns & ns \\
\hline Drug abuse or dependence & ns & ns & ns & ns & ns \\
\hline Attention deficit hyperactive disorder & ns & ns & ns & ns & $2.01(1.34-3.01)$ \\
\hline Any tic disorder & ns & $1.38(1.01-1.89)$ & ns & ns & $1.55(1.14-2.09)$ \\
\hline Tourette syndrome & ns & ns & ns & $0.55(0.34-0.90)$ & ns \\
\hline
\end{tabular}

$\mathrm{ns}=$ non-significant $\mathrm{OR}=$ Odds ratio $\mathrm{CI}=$ confidence interval .

non-paraphilic sexual disorder, somatoform disorders, BDD and tic disorders were only associated with the sexual-religious dimension. These results reinforce the appropriateness of evaluating the "aggressive obsessions and related compulsions" and the "sexualreligious obsessions and related compulsions" as separate constructs (Rosario-Campos et al., 2006).

Aggressive and sexual-religious (or "taboo") thoughts have been previously associated with anxiety disorders (Hasler et al. 2005), depressive disorders (Hasler et al., 2005; Nestadt et al., 2009; De la Cruz et al., 2013) and drug dependence (Brakoulias et al., 2013). The panic-agoraphobia association with these obsessions is understandable, since severe anxious symptoms may occur in OCD patients presenting with excessive fear of being assaulted, but also fear of losing control over one's own 'forbidden' yet unwanted sexual or aggressive impulses. In addition, fear of losing control is a frequent symptom during panic attacks and agoraphobic manifestations may partially overlap with OCD avoidant behaviors, such as fear of losing control over one's impulses in several situations. Nestadt et al. (2009) subdivided 706 individuals with OCD in three groups, according to their comorbidity profile and described an OCD 'comorbid affective-related' class that included predominantly females, with high scores on the 'taboo factor' and common panic and affective disorders. The association between panic disorder/agoraphobia and separation anxiety disorder have been previously described (Rucci et al., 2009; Roberson-Nay et al., 2012) and, in a study comparing OCD patients with and without comorbid separation anxiety disorder (Mroczkowski et al., 2011), the former presented significantly higher rates of panic disorder and agoraphobia.

Interestingly, PTSD was correlated with the aggressive dimension, suggesting that intrusive thoughts related to violence may be related to prior trauma exposure. Aggressive thoughts may be either a consequence of this exposure (i.e., overestimation of threat after a real experience of violence) or a vulnerability factor for developing PTSD when exposed to a traumatic event (Shavitt et al., 2010; Fontenelle et al., 2012). Future studies, with a longitudinal design, are warranted to further investigate the association between aggressive thoughts and PTSD. Of note, PTSD and skin- 
picking remained associated with the aggressive (and hoarding) dimension, in line with the study by Zlotnick et al. (1999), in which self-mutilation/aggressive behaviors were associated with PTSD, even controlling for personality disorder comorbidity. Therefore, the relationships among comorbid disorders should be taken into consideration, since some of them may share common etiological factors or a disorder may increase the vulnerability for the development of subsequent disorders. As previously described, in De Mathis et al. (2013), the comorbidity profile of OCD patients varied according with the first appearing psychopathological manifestation: PTSD and depression were more likely to occur when separation anxiety disorder was the first diagnosis, substance use disorders when ADHD was the initial problem, and OC spectrum disorders when tic disorders occurred firstly.

Mood disorders (major depression, dysthymia and bipolar I disorder) were independent correlates only of the sexual-religious dimension. Depressive symptoms were also associated with sexual obsessions in 383 children and adolescents with OCD (De la Cruz et al., 2013). Again, within the limitations of a cross-sectional design, sexual-religious thoughts may be particularly related to guilt feelings and secondary depressive symptoms.

Social phobia and BDD present partial phenomenological overlap with the OCD avoidant behaviors of social situations frequently associated with sexual-religious thoughts and impulses. Notably, impulse-compulsive non-paraphilic sexual disorder was almost 13 times more frequent among patients presenting with sexual-religious obsessions. This may be due to actual excessive and 'unacceptable' sexual behaviors or may reflect the patients' obsessive doubts and/or dysfunctional beliefs that obsessive sexual thoughts are morally equivalent to sexual behaviors (thought-act fusion). Trying to avoid 'unacceptable' sexual thoughts/images typically leads to an exacerbation of these unwanted thoughts and worsening of symptoms, which may also account for the associations with depression.

We also found the sexual/religious dimension to be associated with bipolar I disorder. Given that reports have suggested a negative association between manic episodes and OCD symptoms (e.g., Amerio et al., 2014), our findings might be better interpreted as evidence of a more specific relationship between bipolar depression and the sexual-religious dimension. Indeed, sexual/religious OCD symptoms were also associated with major depressive disorder (unipolar depression); bipolar depression has been reported to be more clearly associated with dysfunctional cognitive attitudes than unipolar depression (Batmaz et al., 2013). Therefore, normative sexual/religious thoughts may vary from being particularly unacceptable during an episode of bipolar depression (thus prompting the emergence of OCD symptoms) to being acceptable and valued during a manic state, thus negatively associated with OCD symptoms. Greater impulsivity may also lead patients with tic disorders to perceive sexual/religious thoughts in a more threatening way, thus maximizing the importance of thoughts and leading to an overrepresentation of symptoms in these patients.

Although major depression, dysthymia, PTSD, ADHD and tic disorders were associated with the symmetry-ordering dimension in the bivariate analysis, no comorbidity remained significantly associated with this dimension in the logistic regression. This may be due to the low prevalence of some comorbidities that had been predicted as possibly related to this dimension (e.g., bipolar disorder, alcohol dependence, bulimia nervosa) - type 2 error - or to the adjustment for the lifetime occurrence of all other dimensions. The symmetry-ordering dimension has been primarily linked to the hoarding dimension in OCD (Samuels et al., 2002; Fontenelle et al., 2004; Storch et al., 2007) and the latter remained associated, for example, with ADHD (Hartl et al., 2005; Tolin and Villavicencio, 2011; Hacker et al., 2012; Frank et al., 2014), tic disorders (Miguel et al., 1997; Coffey et al., 1998; Samuels et al., 2007, 2014) and
ADHD (Hartl et al., 2005; Tolin and Villavicencio, 2011; Hacker et al., 2012; Frank et al., 2014). Therefore, previous associations might have been confounded by co-occurring hoarding symptoms. In contrast to some dimensions that present a phenomenological overlap with particular comorbid disorders (e.g., contamination dimension and generalized or health anxiety), attenuated relations with the symmetry-ordering dimension may exist given its limited overlap with other comorbid conditions. This dimension may also be more related to personality pathology - particularly cluster B personality disorders (Ha et al., 2004) - an aspect that was not investigated in the present study.

The association between the contamination-cleaning dimension and hypochondriasis may be due to the common phenomenological aspect of excessive health anxiety. This is not a surprising finding, since somatic obsessions and hypochondriacal preoccupations often present with limited insight, and several contamination concerns involve the possibility of contracting a specific illness (e.g., AIDS). For instance, it is a short step from fearing being contaminated with an infectious agent to believing that a physician has not identified this very specific agent in one's blood. The negative association between the contaminationcleaning dimension and tic disorders has been previously reported (De Mathis et al., 2009).

Several associations between the hoarding dimension and specific disorders that we observed in this study have been previously reported in the literature, including depressive disorders (Winsberg et al., 1999; Coles et al., 2003; Frost et al., 2011; Tolin and Villavicencio, 2011; Hall et al., 2013), specific phobia, PTSD (LaSalle-Ricci et al., 2006), ICD in general (Winsberg et al., 1999; Grisham et al., 2007; Tolin and Villavicencio, 2011; Timpano et al., 2014), compulsive buying (Winsberg et al., 1999; Mueller et al., 2009; Frost et al., 2009, 2011; Bulli et al., 2014), skin picking (Samuels et al., 2002), ADHD (Hartl et al., 2005; Tolin and Villavicencio, 2011; Hacker et al., 2012; Frank et al., 2014) and tic disorders (Samuels et al., 2007, 2014). The association with impulsive-compulsive internet use may be related to being frequently socially isolated and sometimes housebound, and maybe also with cybernetic hoarding (e.g., electronic files and e-mails). Some negative findings may be due to the observed low prevalence rates of the conditions (e.g., kleptomania, substance use and bipolar disorders) and/or to the statistical adjustment for other dimensions.

\subsection{Limitations}

Some limitations of this study should to be acknowledged. The cross-sectional design does not permit causal inferences and the age of onset of the disorders was not considered. The generalization of the present findings to other clinical or community samples should be made with caution, since the participants were attending tertiary mental health services and can present with more severe OCD and frequent comorbid disorders. Personality disorders were not investigated and, despite the large sample, some comorbidities were rare (e.g., bipolar disorder, most impulse-control and eating disorders, drug use), perhaps limiting the study power to detect significant associations.

\section{Conclusions}

In this study, different symptom dimensions presented specific associations with lifetime comorbidities and this may have important etiological and clinical implications. The aggressive dimension was associated with PTSD, separation anxiety disorder, any ICD and skin picking; the sexual-religious dimension with mood disorders, panic disorder/agoraphobia, social phobia, 
separation anxiety disorder, non-paraphilic sexual disorder, any somatoform disorder, BDD and tic disorders; the contaminationcleaning dimension with hypochondriasis; and the hoarding dimension with depressive disorders, specific phobia, PTSD, ICDs (compulsive buying, skin picking, internet use), ADHD and tic disorders.

These specific associations between OCD symptom dimensions and comorbid conditions may be partially due to a phenomenological overlap, to common etiological factors and also to mutual influences, since there is evidence of reciprocal and sequential relations between most comorbid forms of psychopathology, although the mechanisms that mediate these links remain to be explained (Cerdá et al., 2008). Therefore, despite the cross-sectional design, this study sheds some light on the complex mosaic of OCD phenomenology that includes considerably different symptoms and co-occurring disorders. Also, this attempt to disentangle the OCD phenotype by looking at individual symptom dimensions in relation to specific comorbidities (i.e. OCD internal and external boundaries) may stimulate new investigations on the underlying pathogenic mechanisms that probably involve diverse genetic and environmental factors, and the interaction between them (Grisham et al., 2008).

Defining more specific phenotypical presentations also has clinical relevance, as they may influence OCD outcome, including the developmental trajectory of other comorbid conditions, helpseeking, adherence and response to treatment. Nevertheless, more studies - particularly with prospective designs - are certainly needed to further understand the temporal sequence of comorbid disorders in OCD according to its symptom dimensions, as well as the influence of specific comorbidities on OCD phenomenology, course and prognosis.

\section{Conflict of interests}

None.

\section{References}

Adam, Y., Meinlschmidt, G., Gloster, A.T., Lieb, R., 2012. Obsessive-compulsive disorder in the community: 12-month prevalence, comorbidity and impairment. Soc. Psychiatry Psychiatr. Epidemiol. 47 (3), 339-349.

Amerio, A., Odone, A., Liapis, C.C., Ghaemi, S.N., 2014. Diagnostic validity of comorbid bipolar disorder and obsessive-compulsive disorder: a systematic review. Acta Psychiatr. Scand. 129 (5), 343-358.

Baer, L., 1994. Factor analysis of symptoms subtypes of obsessive-compulsive disorder and their relation to personality and tic disorders. J. Clin. Psychiatry 55 (Suppl. 3), S18-S23.

Batmaz, S.L., Kaymak, S.U., Soygur, A.H., Ozalp, E., Turkcapar, M.H., 2013. The distinction between unipolar and bipolar depression: a cognitive theory perspective. Compr. Psychiatry 54 (7), 740-749.

Bloch, M.H., Landeros-Weisenberger, A., Rosario, M.C., Pittenger, C., Leckman, J.F., 2008. Meta-analysis of the symptom structure of obsessive-compulsive disorder. Am. J. Psychiatry 165, 1532-1542.

Borges, C.P., Meyer, E., Ferrão, Y.A., Souza, F.P., Sousa, M.B., Cordioli, A.V., 2011. Cognitive-behavioral group therapy versus sertraline for obsessive-compulsive disorder: five-year follow-up. Psychother. Psychosom. 80 (4), 249-250.

Brakoulias, V., Starcevic, V., Berle, D., Milicevic, D., Moses, K., Hannan, A., Sammut, P., Martin, A., 2013. The characteristics of unacceptable/taboo thoughts in obsessive-compulsive disorder. Compr. Psychiatry 54 (7), 750-757.

Bulli, F., Melli, G., Carraresi, C., Stopani, E., Pertusa, A., Frost, R.O., 2014. Hoarding behaviour in an Italian non-clinical sample. Behav. Cogn. Psychother. 42 (3), 297-311.

Caspi, A., Vishne, T., Sasson, Y., Gross, R., Livne, A., Zohar, J., 2008. Relationship between childhood sexual abuse and obsessive-compulsive disorder: case control study. Isr. J. Relat. Sci. 45 (3), 177-182.

Cerdá, M., Sagdeo, A., Galea, S., 2008. Comorbid forms of psychopathology: key patterns and future research. Epidemiol. Rev. 30, 155-177.

Chacon, P.N., Rosario-Campos, M.C., Pauls, D.L., Hounie, A.G., Curi, M., Akkerman, F. Simakoburo, F.H., de Mathis, M.A., Lopes, A.C., Hasler, G., Miguel, E.C., 2007. Obsessive-compulsive symptoms in sibling pairs concordant for obsessivecompulsive disorder. Am. J. Med. Gen. 144B (4), 551-555.
Chakraborty, V., Cherian, A.V., Math, S.B., Venkatasubramanian, G., Thennarasu, K., Mataix-Cols, D., Reddy, Y.C., 2012. Clinically significant hoarding in obsessivecompulsive disorder: results from an Indian study. Compr. Psychiatry 53 (8), 1153-1160.

Coffey, B.J., Miguel, E.C., Biederman, J., Baer, L., Rauch, S.L., O'Sullivan, R.L., Savage, C. R., Phillips, K., Borgman, A., Green-Leibovitz, M.I., Moore, E., Park, K.S., Jenike, M. A., 1998. Tourette disorder with and without obsessive-compulsive disorder in adults: are they different? J. Nerv. Ment. Dis. 186 (4), 201-206.

Coles, M.E., Frost, R.O., Heimberg, R.G., Steketee, G., 2003. Hoarding behaviors in a large college sample. Behav. Res. Ther. 41 (2), 179-194.

De la Cruz, L.F., Barrow., F., Bolhuis, K., Krebs, G., Volz, C., Nakatani, E., Heyman, I., Mataix-Cols, D., 2013. Sexual obsessions in pediatric obsessive-compulsive disorder : clinical characteristics and treatment outcomes. Depression Anxiety 30, $732-740$.

De Mathis, M.A., Diniz, J.B., Shavitt, R.G., Torres, A.R., Ferrão, Y.A., Fossaluza, V., Pereira, C., Miguel, E.C., do Rosario, M.C., 2009. Early onset obsessive-compulsive disorder with and without tics. CNS Spectr. 14 (7), 362-370.

De Mathis, M.A., Diniz, J.B., Hounie, A.G., Shavitt, R.G., Fossaluza, V., Ferrão, Y.A., et al., 2013. Trajectory in obsessive-compulsive disorder comorbidities. Eur. Neuropsychopharmacol. 23 (7), 594-601.

Denys, D., Tenney, N., Van Megen, H.J., De Geus, F., Westenberg, H.G., 2004. Axis I and II comorbidity in a large sample of patients with obsessive-compulsive disorder. J. Affect. Disord. 80, 155-162.

Ferrão, Y.A., Shavitt, R.G., Bedin, N.R., Mathis, M.A., Lopes, A.C., Fontenelle, L.F., et al., 2006. Clinical features associated to refractory obsessive compulsive disorder. J. Affect. Disord. 94, 199-209.

First, M.B., Spitzer, R.L., Gibbonm, M., Williams, J.B.W., 1995. Structured Clinical Interview for DSM-IV-TR Axis I Disorders, Research Version, Patient Edition. (SCID-I/P). Biometrics Research: New York State Psychiatric Institute, New York.

First, M.B., 2004. Structural Clinical Interview for DSM-IV-TR Impulse Control Disorders Not Elsewhere Classified (SCID-TCIm). New York State Psychiatric Institute: Biometrics Research Department.

Fineberg, N.A., Hengartner, M.P., Bergbaum, C., Gale, T., Rössler, W., Angst, J., 2013. Lifetime comorbidity of obsessive-compulsive disorder and sub-threshold obsessive-compulsive symptomatology in the community: impact, prevalence, socio-demographic and clinical characteristics. Int. J. Psychiatry Clin. Pract. 17 (3), 188-196.

Fontenelle, L.F., Mendlowicz, M.V., Soares, I.D., Versiani, M., 2004. Patients with obsessive-compulsive disorder and hoarding symptoms: a distinctive clinical subtype? Compr. Psychiatry 45 (5), 375-383.

Fontenelle, L.F., Cocchi, L., Harrison, B.J., Shavitt, R.G., do Rosário, M.C., Ferrão, Y.A., et al., 2012. Towards a post-traumatic subtype of obsessive compulsive disorder. J. Anxiety Disord. 26, 377-383.

Frank, H., Stewart, E., Walther, M., Benito, K., Freeman, J., Conelea, C., Garci, A., 2014 Hoarding behavior among young children with obsessive-compulsive disorder. J. Obs. Compuls. Relat. Disord. 3 (1), 6-11.

Frost, R.O., Tolin, D.F., Steketee, G., Fitch, K.E., Selbo-Bruns, A., 2009. Excessive acquisition in hoarding. J. Anxiety Disord. 23, 632-639.

Frost, R.O., Steketee, G., Tolin, D.F., 2011. Comorbidity in hoarding disorder. Depression Anxiety 28 (10), 876-884.

Fullana, M.A., Tortella-Feliu, M., Caseras, X., Taberner, J., Torrubia, R., Mataix-Cols, D., 2007. Temporal stability of obsessive-compulsive symptom dimensions in an undergraduate sample: a prospective 2-year follow-up study. Behav. Modif. 31 (6), 815-824.

Fullana, M.A., Mataix-Cols, D., Caspi, A., Harrington, H., Grisham, J.R., Moffitt, T.E. et al., 2009. Obsessions and compulsions in the community: prevalence, interference, help-seeking, developmental stability, and co-occurring psychiatric conditions. Am. J. Psychiatry 166, 329-336.

Gomes de Alvarenga, P., de Mathis, M.A., Dominguez Alves, A.C., do Rosário, M.C., Fossaluza, V., Hounie, A.G., Miguel, E.C., Torres, A.R., 2012. Clinical features of tic-related obsessive-compulsive disorder: results from a large multicenter study. CNS Spectr. 17 (2), 87-93.

Goodman, W.K., Price, L.H., Rasmussen, S.A., Mazure, C., Delgado, P., Heninger, G.R., Charney, D.S., 1989. The yale-brown obsessive-compulsive scale: validity. Arch. Gen. Psychiatry 41, 1012-1016.

Grant, J.E., Pinto, A., Gunnip, M., Mancebo, M.C., Eisen, J.L., Rasmussen, S.A., 2006. Sexual obsessions and clinical correlates in adults with obsessive-compulsive disorder. Compr. Psychiatry 47, 325-329.

Grisham, J.R., Anderson, T.M., Sachdev, P.S., 2008. Genetic and environmental influences on obsessive-compulsive disorder. Eur. Arch. Psychiatry Clin. Neurosci. 258 (2), 107-116.

Grisham, J.R., Brown, T.A., Savage, C.R., Steketee, G., Barlow, D.H., 2007. Neuropsychological impairment associated with compulsive hoarding. Behav. Res. Ther. 45 (7), 1471-1483.

Ha, T.H., Youn, T., Rho, K.S., Kim, M.S., Kwon, J.S., 2004. Symptom dimensions of obsessive-compulsive disorder and their relation to comorbid personality pathology. J. Korean Neuropsychiatr. Assoc. 43 (1), 46-53.

Hacker, L.E., Park, J.M., Timpano, K.R., Cavitt, M.A., Alvaro, J.L., Lewin, A.B., Murphy, T.K., Storch, E.A., 2012. Hoarding in children with ADHD. J. Atten. Disord., August 24. [Epub ahead of print]

Hall, B.J., Tolin, D.F., Frost, R.O., Steketee, G., 2013. An exploration of comorbid symptoms and clinical correlates of clinically significant hoarding symptoms. Depression Anxiety 30 (1), 67-76.

Harrison, B.J., Pujol, J., Cardoner, N., Deus, J., Alonso, P., López-Solà, M., ContrerasRodríguez, O., Real, E., Segalàs, C., Blanco-Hinojo, L., Menchon, J.M., SorianoMas, C., 2013. Brain corticostriatal systems and the major clinical symptom 
dimensions of obsessive-compulsive disorder. Biol. Psychiatry 73 (4), 321-328.

Hartl, T.L., Duffany, S.R., Allen, G.J., Steketee, G., Frost, R.O., 2005. Relationships among compulsive hoarding, trauma, and attention-deficit/hyperactivity disorder. Behav. Res. Ther. 43 (2), 269-276.

Hasler, G., LaSalle-Ricci, V.H., Ronquillo, J.G., Crawley, S.A., Cochran, L.W., Kazuba, D., Greenberg, B.D., Murphy, D.L., 2005. Obsessive-compulsive disorder symptom dimensions show specific relationships to psychiatric comorbidity. Psychiatry Res. 135 (2), 121-132.

Hasler, G., Pinto, A., Greenberg, B.D., Samuels, J., Fyer, A.J., Pauls, D., et al., 2007. Familiality of factor analysis-derived YBOCS dimensions in OCD-affected sibling pairs from the OCD Collaborative Genetics Study. Biol. Psychiatry 61, 617-625.

Hofmeijer-Sevink, M.K., Van Oppen, P., van Megen, H.J., Batelaan, N.M., Cath, D.C., der Wee, N.J.A., 2013. Clinical relevance of comorbidity in obsessive-compulsive disorder: The Netherlands OCD Association study. J. Affect. Disord. 150 (3), 847-854.

Jakubovski, E., Diniz, J.B., Valerio, C., Fossaluza, V., Belotto-Silva, C., Gorenstein, C., Miguel, E.C., Shavitt, R.G., 2013. Clinical predictors of long-term outcome in obsessive-compulsive disorder. Depression Anxiety 30 (8), 763-772.

Janowitz, D., Garbe, H.J., Ruhrmann, S., Ettel, S., Buhtz, F., Hechrein, A., et al., 2009. Early onset obsessive-compulsive disorder and associated comorbidity. Depression Anxiety 26 (11), 1012-1017.

Kalra, H., Trivedi, J.K., Dalal, P.K., Sinha, P.K., Allet, J.L., 2008. Uncomplicated and complicated obsessive-compulsive disorder: an exploratory study from India. Compr. Psychiatry 49, 51-54.

Landeros-Weisenberger, A., Bloch, M.H., Kelmendi, B., Wegner, R., Nudel, J., Dombrowski, P., Pittenger, C., Krystal, J.H., Goodman, W.K., Leckman, J.F., Coric, V., 2010. Dimensional predictors of response to SRI pharmacotherapy in obsessive-compulsive disorder. J. Affect. Disord. 121 (1-2), 175-179.

LaSalle, V.H., Cromer, K.R., Nelson, K.N., Kazuba, D., Justement, L., Murphy, D.L., 2004. Diagnostic interview assessed neuropsychiatric disorder comorbidity in 334 individuals with obsessive-compulsive disorder. Depression Anxiety 19, $163-173$.

LaSalle-Ricci, V.H., Arnkoff, D.B., Glass, C.R., Crawley, S.A., Ronquillo, J.G., Murphy, D. L., 2006. The hoarding dimension of OCD: psychological comorbidity and the five-factor personality model. Behav. Res. Ther. 44, 1503-1512.

Leckman, J.F., Grice, D.E., Boardman, J., Zhang, H., Vitale, A., Bondi, C., Alsobrook, J., Peterson, B.S., Cohen, D.J., Rasmussen, S.A., Goodman, W.K., McDougle, C.J., Pauls, D.L., 1997. Symptoms of obsessive-compulsive disorder. Am. J. Psychiatry 154 (7), 911-917.

Lochner, C., Fineberg, N.A., Zohar, J., van Ameringen, M., Juven-Wetzler, A., Altamura, A.C., Cuzen, N.L., Hollander, E., Denys, D., Nicolini, H., Dell'Osso, B., Pallanti, S., Stein, D.J., 2014. Comorbidity in obsessive-compulsive disorder (OCD): a report from the International College of Obsessive-Compulsive Spectrum Disorders (ICOCS). Compr. Psychiatry 55 (7), 1513-1519.

Mataix-Cols, D., Rauch, S.L., Manzo, P.A., Jenike, M.A., Baer, L., 1999. Use of factoranalyzed symptom dimensions to predict outcome with serotonin reuptake inhibitors and placebo in the treatment of obsessive-compulsive disorder. Am. J. Psychiatry 156 (9), 1409-1416.

Mataix-Cols, D., Rosario-Campos, M.C., Leckman, J.F., 2005. A multidimensional model of obsessive-compulsive disorder. Am. J. Psychiatry 162, 228-238.

Mataix-Cols, D., Marks, I.M., Gresit, J.H., Kobak, K.A., Baer, L., 2002. Obsessivecompulsive symptom dimension as predictor of compliance with and response to behavior therapy: results from a controlled trial. Psychother. Psychosom. 71 (5), 255-262.

Mataix-Cols, D., Wooderson, S., Lawrence, N., Brammer, M.J., Speckens, A., Phillips, M.L., 2004. Distinct neural correlates of washing, checking, and hoarding symptoms dimensions in obsessive-compulsive disorder. Arch. Gen. Psychiatry 61 (6), 564-576.

McGuire, J.F., Crawford, E.A., Park, J.M., Storch, E.A., Murphy, T.K., Larson, M.J., Lewin, A.B., 2014. Neuropsychological performance across symptom dimensions in pediatric obsessive compulsive disorder. Depression Anxiety 31 (12), 988-996.

Miguel, E.C., Baer, L., Coffey, B.J., Rauch, S.L., Savage, C.R., O'Sullivan, R.L., Phillips, K., Moretti, C., Leckman, J.F., Jenike, M.A., 1997. Phenomenological differences appearing with repetitive behaviours in obsessive-compulsive disorder and Gilles de la Tourette syndrome. Br. J. Psychiatry 170, 140-145.

Mroczkowski, M.M., Goes, F.S., Riddle, M.A., Grados, M.A., Bienvenu 3rd, O.J., Greenberg, B.D., Fyer, A.J., McCracken, J.T., Rauch, S.L., Murphy, D.L., Knowles, J. A., Piacentini, J., Cullen, B., Rasmussen, S.A., Geller, D.A., Pauls, D.L., Liang, K.Y., Nestadt, G., Samuels, J.F., 2011. Separation anxiety disorder in OCD. Depression Anxiety 28 (3), 256-262.

Miguel, E.C., Ferrão, Y.A., Rosário, M.C., de Mathis, M.A., Torres, A.R., Fontenelle, L.F., et al., 2008. The Brazilian Research Consortium on Obsessive-Compulsive Spectrum Disorders (CTOC): recruitment, assessment instruments, methods for the development of multicenter collaborative studies and preliminary results. Rev. Bras. Psiquiatr. 30 (3), 187-198.

Mueller, A., Mitchell, J.E., Crosby, R.D., Glaesmer, H., de Zwann, M., 2009. The prevalence of compulsive hoarding and its association with compulsive buying in a German population-based sample. Behav. Res. Ther. 47 (8), 705-709.

Nestadt, G., Di, C.Z., Riddle, M.A., Grados, M.A., Greenberg, B.D., Fyer, A.J., McCracken, J.T., et al., 2009. Obsessive-compulsive disorder: subclassification based on co-morbidity. Psychol. Med. 39 (9), 1491-1501.

Pigott, T.A., L'Heureux, F., Dubbert, B., Bernstein, S., Murphy, D.L., 1994. Obsessive compulsive disorder: comorbid conditions. J. Clin. Psychiatry 55 (Suppl.), S15-S27.

Raffin, A.L., Guimarães Fachel, J.M., Ferrão, Y.A., Pasquoto de Souza, F., Cordioli, A.V., 2009. Predictors of response to group cognitive-behavioral therapy in the treatment of obsessive-compulsive disorder. Eur. Psychiatry 24 (5), 297-306.

Roberson-Nay, R., Eaves, L.J., Hettema, J.M., Kendler, K.S., Silberg, J.L., 2012. Childhood separation anxiety disorder and adult onset panic attacks share a common genetic diathesis. Depression Anxiety 29 (4), 320-327.

Rosario-Campos, M.C., Miguel, E.C., Quatrano, S., Chacon, P., Ferrão, Y., Findley, D., Katsovich, L., Scahill, L., King, R.A., 2006. The Dimensional Yale-Brown Obsessive-Compulsive Scale (DY-BOCS): an instrument for assessing obsessivecompulsive symptom dimensions. Mol. Psychiatry 11 (5), 495-504.

Rucci, P., Miniati, M., Oppo, A., Mula, M., Calugi, S., Frank, E., Shear, M.K., Mauri, M., Pini, S., Cassano, G.B., 2009. The structure of lifetime panic-agoraphobic spectrum. J. Psychiatr. Res. 43 (4), 366-379.

Rufer, M., Grothusen, A., Mass, R., Peter, H., Hand, I., 2005. Temporal stability of symptom dimensions in adult patients with obsessive-compulsive disorder. J. Affect. Disord. 88 (1), 99-102.

Ruscio, A.M., Stein, D.J., Chiu, W.T., Kessler, R.C., 2010. The epidemiology of obsessive-compulsive disorder in the National Comorbidity Survey Replication. Mol. Psychiatry 15, 53-63.

Samuels, J., Shugart, Y.Y., Grados, M.A., Willour, V.L., Bienvenu, J., Greenberg, B.D., et al., 2007. Significant linkage to compulsive hoarding on cromossome 14 in families with obsessive-compulsive disorder: results from the OCD Collaborative Genetics Study. Am. J. Psychiatry 164, 493-499.

Samuels, J., Bienvenu, O.J., Riddle, M.A., Cullen, B.A., Grados, M.A., Ling, K.Y., HoehnSaric, R., Nestadt, G., 2002. Hoarding in obsessive-compulsive disorder: results from a case-control study. Behav. Res. Ther. 40, 517-528.

Samuels, J., Grados, M.A., Riddle, M.A., Bienvenu, O.J., Goes, F.S., Cullen, B., et al., 2014. Hoarding in children and adolescents with obsessive-compulsive disorders. J. Obs. Compuls. Relat. Dis. 3 (4), 325-331.

Shavitt, R.G., Valério, C., Fossaluza, V., da Silva, E.M., Cordeiro, Q., Diniz, J.B., BelottoSilva, C., Cordioli, A.V., Mari, J., Miguel, E.C., 2010. The impact of trauma and post-traumatic stress disorder on the treatment response of patients with obsessive-compulsive disorder. Eur. Arch. Psychiatry Clin. Neurosci. 260 (2), 91-99.

Stata Corporation, 2011. Stata Statistical Software. Release 12.0. College Station, Stata Corporation, TX.

Storch, A.L., Lack, C.W., Merlo, L., Geffken, G.R., Jacob, M.L., Murphy, T.K., Goodman W.K., 2007. Clinical features of children and adolescents with obsessive-compulsive disorder and hoarding symptoms. Compr. Psychiatry 48, 313-318.

Timpano, K.R., Shaw, A.M., Cougle, J.R., Fitch, K.E., 2014. A multifaceted assessment of emotional tolerance and intensity in hoarding. Behav. Ther. 45 (5), 690-699.

Tolin, D.F., Villavicencio, A., 2011. Inattention, but not OCD, predicts the core features of hoarding disorder. Behav. Res. Ther. 49 (2), 120-125.

Torres, A.R., Prince, M.J., Bebbington, P.E., Bhugra, D., Brugha, T.S., Farrell, M., et al., 2006. Obsessive-compulsive disorder: prevalence, comorbidity, impact, and help-seeking in the British National Psychiatric Morbidity Survey of 2000. Am. J. Psychiatry 163, 1978-1985.

Torres, A.R., Ramos-Cerqueira, A.T.A., Ferrão, Y.A., Fontenelle, L.F., Rosário, M.C., Miguel, E.C., 2011. Suicidality in obsessive-compulsive disorder: prevalence and relation to symptom dimensions and comorbid conditions. J. Clin. Psychiatry 72 (1), 17-26.

Tükel, R., Polat, A., Ozdemir, O., Aksüt, D., Türksoy, N., 2002. Comorbid conditions in obsessive-compulsive disorder. Compr. Psychiatry 43, 204-209.

van den Heuvel, O.A., Remijnse, P.L., Mataix-Cols, D., Vrenken, H., Groenewegen, H. J., Uylings, H.B., van Balkom, A.J., Veltman, D.J., 2009. The major symptom dimensions of obsessive-compulsive disorder are mediated by partially distinct neural systems. Brain 132 (Pt. 4), 853-868.

Via, E., Cardoner, N., Pujol, J., Alonso, P., López-Solà, M., Real, E., Contreras-Rodríguez, O., Deus, J., Segalàs, C., Menchón, J.M., Soriano-Mas, C., Harrison, B.J., 2014. Amygdala activation and symptom dimensions in obsessive-compulsive disorder. Br. J. Psychiatry 204 (1), 61-68.

Wheaton, M., Timpano, K.R., Lassalle-Ricci, V.H., Murphy, D., 2008. Characterizing the hoarding phenotype in individuals with OCD: associations with comorbidity, severity and gender. J. Anxiety Disord. 22 (2), 243-252.

Winsberg, M.E., Cassic, K.S., Koran, L.M., 1999. Hoarding in obsessive-compulsive disorder: a report of 20 cases. J. Clin. Psychiatry 60 (9), 591-597.

Zlotnick, C., Mattia, J.I., Zimmerman, M., 1999. Clinical correlates of self-mutilation in a sample of general psychiatric patients. J. Nerv. Ment. Dis. 187 (5), 296-301. 